\title{
Chapter 11 \\ Experiential Sociometry Practice and Safety Structures with Groups
}

\begin{abstract}
This chapter is devoted to the description and depiction of action sociometry processes and safety structures in group settings, especially in group therapy. Each of these is presented with considerations to theory, safety, and multiple examples of structured prompts for their application in diverse social work group settings. Experiential sociometry processes outlined include the use of small groups, spectrograms, locograms, floor checks, step-in sociometry, hand-on-shoulder soicograms, and the circle of strength safety structure. These action-based processes can be modified for use in any group setting to enliven the group experience.
\end{abstract}

Keywords Experiential sociometry $\cdot$ Spectrogram $\cdot$ Locogram $\cdot$ Floor check Sociogram $\cdot$ Circle of strengths

A previous chapter explored sociometry as a theoretical system and a research methodology for exploring and understanding small groups, social networks, and society (see Chap. 5). This chapter is entirely devoted to the clinical practice of sociometry within group work and group therapy. In addition to five commonly used experiential sociometric processes (small groups, spectrograms, step-in sociometry, hands-on-shoulder sociometry, and locograms), this chapter will also present the circle of strength safety structure from the Therapeutic Spiral Model and the sociometric floor check instrument from the Relational Trauma Repair Model. While the emphasis of this chapter will be on in-person groups, content on adapting these methods for teletherapy will also be introduced throughout the text.

\subsection{Clinical Applications of Sociometry}

The clinical applications of sociometry include a variety of pen-to-paper activities that explore an individual's social atom or social network, or experiential action structures that explore the series of attractions, repulsions, similarities, and differences within the group (Hale, 1981, 2009). In the context of this discussion of 
group psychotherapy, clinical applications for groups will be primarily emphasized-though sociometric tools are also used in individual settings (see Chap. 16), community work (see Chap. 18), and education (see Chap. 20).

There are numerous other commonly used experiential sociometric group processes that are employed in clinical settings including dyadic or triadic sharing, spectrograms, locograms, floor checks, step-in sociometry, and hands-on-shoulder sociometry (Dayton, 2005, 2014, 2015; Giacomucci, 2017, 2018a, 2019, 2020b; Giacomucci et al., 2018; Hale, 1981, 2009; Hudgins \& Toscani, 2013). Each of these processes can be modified with content appropriate for any population or chosen topic. In terms of clinical uses of sociometry, these sociometric tools often stand on their own as multidimensional action-based group processes that provide the group with an avenue to discover and enrich their connections with each other. Sociometry can also be employed with an objective of recognizing shared identity between group members including membership to privileged or oppressed groups (Nieto, 2010). These same sociometric action structures frequently serve as a group warmup exercise before conducting a psychodrama but can be used in the beginning, middle, or ending stages of a group. Each of these experiential sociometry structures can be used for group assessment, exploration, intervention, and evaluation.

Regardless of which sociometry tool is being utilized, it is useful for both the group and the facilitator if a clinical map is employed. To cultivate an experience of safety, vulnerability, containment, and warming-up, it can help to select criteria for prompts while adhering to the three clinical maps for trauma (Chesner, 2020; Courtois \& Ford, 2016; Herman, 1997; Giacomucci, 2018b; Hudgins \& Toscani, 2013). Initial prompts would be simple and strengths-based with the goal of facilitating connection. The next prompt(s) invite the group to share deeper with a focus on difficult emotions, defense mechanisms, trauma, loss, addiction, or mental illness. And to finish, making an offer of prompt(s) that facilitate or engage with meaning-making, integration, future projection, and post-traumatic growth. Essentially, this clinical map starts with positive prompts, moves into negative prompts, and ends with positive prompts again. This process reflects the process of slowly moving in and out of difficult emotional content — called pendulation and titration described by trauma experts (Courtois \& Ford, 2016; Herman, 1997; Levine, 2010; Shapiro, 2018; van der Kolk, 2014). While providing containment, direction, and safety for the larger group process, this practice may also help clients internalize a sense of containment, self-efficacy, and safety related to their trauma (Giacomucci \& Marquit, 2020).

\subsection{Dyads, Triads, and Small Groups}

Moreno often wrote of the importance of dyadic connection within groups and suggested that group cohesion was a function of the number of reciprocated mutual choices within the group (Hale, 2009; Moreno, 1934). Consequently, the use of dyadic sharing and breaking the larger group into smaller pairs can help facilitate interpersonal connection, mutual aid, and overall group cohesion (Giacomucci, 2020b). 
Using dyads, triads, or small breakout groups seems to be especially useful at the early stages of groups and when working with larger groups. Many clients do not feel comfortable sharing in front of large groups-using smaller groups allows for participants to feel safer as they are invited to share with one or two others. This process also mitigates the chances of one participant monopolizing the group discussion and preventing the more reluctant or introverted group members from actively engaging (Olesen et al., 2017). The process of a traditional talk-based group only permits one discussion to take place at a time, but using dyads or small groups allows for multiple discussions to take place concurrently. The nature of small group discussions, especially dyads, invokes a role demand for each participant to respond, attend to, support, and share with their partner - thus engaging in mutual aid. The process of utilizing small group discussion frees up the facilitator to move around the room and listen to or check in with each group. This allows the facilitator to take a less active role and indirectly reveals the facilitator's confidence, trust, and faith in each participant's ability to serve as a therapeutic agent for each other.

The method of choosing configurations of small groups or dyads can be modified depending on the facilitator's intention or the nature of the group. Partners or small groups can be assigned randomly, intentionally chosen by the facilitator, self-selected by participants, or chosen based on prompts. When inviting participants to self-select a partner or break into triads, participants are likely to choose the person(s) physically closest to them. Often, they are already sitting next to the group members that they feel most connected to so this may lead to already cohesive small groups and few new connections. Prompting participants to "partner with someone you do not know well" is a simple way to facilitate dyads ripe with new opportunities to connect.

In terms of the content of the discussion within the small groups, it helps to offer directed prompts related to the nature of the group or the therapeutic goals of the session guided by the aforementioned three-stage clinical map. Generally, 3-5 prompts seem to be sufficient for small group discussions in terms of adequately warming up the group without losing the interest of participants. Below are some examples of prompts following the aforementioned clinical map based on different topics for clinical groups:

\section{Topic: Relationships}

1. Share about one of your favorite relationships.

2. Share about a person who has helped you in some way recently.

3. Share about a relationship that you have difficulty or conflict in.

4. Share how you would a difficult relationship to be different in the future.

5. Share about one way you would like to change your behavior in relationships.

\section{Topic: Emotions}

1. Share a memory that gives you a positive feeling.

2. Share about the emotion that you have most difficulty with.

3. Share about ways you could respond to difficult emotions different in the future.

\section{Topic: Mental Illness}


1. Share about tools you have to cope with symptoms of mental illness.

2. Share about one role model for living with mental illness (personal, societal, archetypal, historical, religious, or even a fantasy character).

3. Share about how your mental illness impacts you.

4. Share about what you image your life would look like in the future without mental illness.

\section{Topic: Trauma}

1. Share about one strength you have that can help you work through your trauma.

2. Share about one step you have already taken toward healing from trauma.

3. Share about one way your trauma has negatively impacted you.

4. Share about one way you would like to grow from your trauma.

5. Share about one step you plan to take in the future toward healing from trauma.

Another method for facilitating dyadic connections within groups is to place cards, objects, or images in the center of the group space and ask participants to choose one based on a guiding prompt. Some prompts might include inviting participants to choose a card that can represent "your strength," "the next step on your journey of healing," "defense mechanism," "your spirituality," "your goal for today," or "your ideal self in the future." Once chosen, group members are instructed to share in dyads about their choice of cards and the symbolic representation. Sharing can be extended with new prompts and/or new partners to facilitate increased connection. This process can be done with any set of cards and used as a warm-up to any topic with various prompts.

The Therapeutic Spiral Model (TSM) utilizes this process of choosing cards to represent the role of the observing ego-sometimes called the compassionate witness or caring observer (Hudgins \& Toscani, 2013; Lawrence, 2015). The observing ego is a concept, borrowed from Freud (1932), which in this context refers to the part of self that can accurately observe self and others without shame, blame, or judgment (Giacomucci, 2018b). In TSM groups, the cards are then placed on the walls of the group room to provide a conscious reminder of this role and its importance. The use of cards to concretize the observing ego, a strength, or any other positive role provides an anchor of safety for the group experience going forward (Giacomucci et al., 2018). The presence of the cards on the walls behind the group offers a sense of being contained as well as an already defined strengths-based role for the psychodrama director to utilize during the group if extra grounding is needed. TSM practitioners primarily use this process to concretize a psychodramatic role, but it is important to note here for the sociometric aspect of using cards or objects to cultivate dyadic sharing at the beginning of a group process. The experience of using small groups or dyads offers participants the opportunity to create new connections, deepen relationships, enhance their sense of belonging, warm up to a topic, and increase overall group cohesion. 


\subsection{Spectrograms}

The spectrogram is essentially a group-as-a-whole assessment tool that allows the facilitator to efficiently gather information about the group while providing participants with the chance to see where they fit in within the group and to connect with each other. A spectrogram is an action-based self-assessment along a spectrum within the room. Facilitation of a spectrogram is done by designating two different objects or two opposite walls of the room to represent the beginning and end of the spectrum. Generally, one side is designated as a $0 / 10$ or $0 \%$ and the opposite side as a $10 / 10$ or $100 \%$ while emphasizing that the imaginary line between the poles includes every possibility between 0 to 10 and $0 \%$ to $100 \%$. Participants are invited to physically place themselves on the imaginary line based on where they believe they belong when considering the prompt(s).

"box[Spectrogram Video] starts"

The following video depicts the use of spectrograms with a live group.

http://www.phoenixtraumacenter.com/spectrograms/

"box[Spectrogram Video] ends"

Some examples of spectrogram prompts following the aforementioned clinical map are depicted below:

\section{Topic: Connection and Loss}

1. How many supportive relationships do you have in your life today?

2. How much loss have you experienced in your life?

3. How well do you think you handle grief?

4. How resilient do you judge yourself to be?

\section{Topic: Addiction and Recovery}

1. How many resources (supports, coping skills, tools, etc.) do you have to help in your recovery?

2. How motivated are you today for your recovery?

3. How much has addiction impacted your life?

4. How hopeful are you for your future in recovery?

\section{Topic: Inpatient Treatment Experiences}

1. How many days have you been in inpatient treatment?

2. How connected do you feel to the inpatient community of clients?

3. How difficult has it been for you to remain in treatment?

4. How much progress do you feel you have made since the day of your entered treatment?

\section{Topic: Stress and Self-care}

1. How many tools for self-care do you have? 
2. How often do you find yourself stressed out?

3. How much tolerance for stress do you judge yourself to have?

4. How many new strategies for coping with stress have you learned from the group?

\section{Topic: Psychoeducation of Trauma}

1. How curious are you to learn more about trauma?

2. How familiar are you with the common ways that trauma impacts people?

a. How aware are you of how trauma has impacted you or your loved ones?

3. How knowledgeable are you in the different treatments and approaches to healing from trauma?

With each new prompt, it is helpful to change the axis of the spectrogram, using different areas in the room. This facilitates more physical movement and prevents participants from staying in the same physical place. Each prompt results in a new configuration of participants along the spectrogram. There are multiple ways to facilitate sharing. The simplest form of sharing is to have the group sharing in groups of 2-3 based on whoever is physically closest to them on the spectrogram. The use of dyadic sharing allows for everyone to share about why they are standing on the spectrogram at the point they are at without taking up too much time. Similarly, participants could be invited to share in clusters of larger groups about where they have chosen to stand on the spectrum. Another option is to ask for group members to share aloud to the group about their placement on the spectrogram. Other nonverbal ways to facilitate spectrogram sharing include with body posture, movement and gestures, or sound.

It is important to consider that the spectrogram is a self-assessment and how that may impact one's choices in the process (Giacomucci et al., 2018). There are spectrogram prompts where it would be advantageous to change the spectrum to a 110 , or even a 5-10 spectrum. For example, when using the prompt "how resilient do you judge yourself to be?" with a group of trauma survivors, it may be empowering to limit the spectrogram to a 5-10 while indicating the belief that everyone in the room is at least of 5/10 in resilience whether they believe it or not. Most of the clients that social workers work with are struggling with trauma, loss, addiction, oppression, mental illness, or other forms of suffering and may have distorted judgments about themselves which will impact their self-assessment in the spectrogram process. When the facilitator notices this happening with a group member, it can be helpful to gently challenge the individual to reconsider or to reflect back to the group member where the facilitator would assess them to be on the spectrum. Another option would be to ask the group to share with the individual about where on the spectrogram they would assess that person to be based on the prompt-thus creating an opportunity for mutual aid.

A spectrogram prompt results in a distribution of group members along a continuum, sometimes with participants spread out, sometimes with clusters of participants in different places, and occasionally with isolates on the high or low 
end of the spectrum. When a prompt results in an obvious isolate on the spectrogram, it can be helpful to explore that person's experience at the group level. If they are an outlier on the higher end of a strengths-based prompt, they likely have important information that they could share to help the rest of the group. If they are an outlier on the lower end of a positive prompt, they might have questions about how to gain more understanding or competence related to the criteria. It can also be helpful to invite group members to raise their hands if they can remember a time in the past when they were at that point on the spectrogram and offer suggestions or identification with the person who is currently an outlier. A psychodramatist might also direct role reversals between group members on the spectrogram to have them explore what it is like to be at various spots. When participants have mobility limitations, the process can be modified by using an object to represent participants on the spectrogram or by having participants raise their hands to varying degrees (10/10 is as high as you can raise your hand; 0/10 is placing your hand on your lap) (Simmons, 2017). Spectrograms can also be easily modified for use during teletherapy or online teaching sessions by using the top and bottom of each participant's video feed as the top and bottom of the spectrum. Participants are simply instructed to place their hand along the vertical spectrum of their camera window to indicate where on the spectrogram they are.

Spectrograms allow the facilitator to meet the group where they are at in terms of their warm-up to a specific topic or their understanding of the topic. They are especially useful in psychoeducational sessions, training workshops, classrooms, and supervision groups to assess the knowledge level of participants at the start of the session. This gives the facilitator the chance to change the content of the session to meet the learning needs of the group (Giacomucci, 2018a). Spectrograms are also useful for evaluation in that the same spectrogram prompt could be asked at the beginning and end of a session or program or pen-to-paper assessments can be modified into experiential spectrograms (Giacomucci, 2020a).

\subsection{Locograms}

The locogram is a sociometry tool that offers a quick visual group assessment or democratic group vote in an experiential format based on different options or categories. As suggested by the prefix of the term locogram, this process is an exploration of choices using places in the room. While the spectrogram orients itself upon a spectrum, the locogram is oriented based on designated locations in the room. One of the simplest ways to facilitate a locogram is using the four corners of the room to each representing a different choice and asking participants to physically indicate their preference by standing at the corresponding location (Giacomucci, 2020a). Other styles of directing a locogram involve using objects or chairs to represent the various choices. Most locograms offer at least 3 options which usually include an option for "other" - this invites other suggestions from the group. Locograms are useful for quick assessments or group choices in action. They can be used for many purposes 
including choosing a topic, discerning when to take a break, making a group decision, assessing group preference, uncovering similarities, and exploring a group's warm-up.

"box[Floor Check Video] starts"

The following video depicts the use of floor check with a live group.

http://www.phoenixtraumacenter.com/floor-check/

"box[Floor Check Video] ends"

Here are some examples with the bullet points representing the different locations in the room (represented by the corners of the room or objects):

\section{Purpose: Choosing a Topic in an Outpatient Group}

- Anxiety

- Depression

- Trauma

- Other.

Purpose: Discerning What the Group is Warmed up for Next

- Discussion

- More sociometry

- Psychodrama

- Writing activity

- Other.

\section{Purpose: Outpatient Group Decision About Holiday Scheduling Conflict}

- Schedule the session on the holiday

- Cancel the session on the holiday

- Meet a different day that week

- Meet twice as long the following session

- Other.

\section{Purpose: Uncovering Shared Experiences in School}

- I hated school

- I loved school

- I had mixed feelings about school

- Other.

\section{Purpose: Exploring Religious/Spiritual Beliefs}

- Christianity

- Islam

- Judaism

- Buddhism 
- Hinduism

- Non-religious spirituality

- Non-believer

- Shamanic

- Other.

The locogram can be used for a quick group-as-a-whole assessment and group choice, or for an exploration of shared perspective, experience, or identity. When facilitating a locogram, it helps to limit or expand the number of options based on the size of the group. For example, in the final example about religious/spiritual belief systems, the nine offered choices in the locogram would be useful in a large group setting as it would facilitate connection, but in a small group it could lead to multiple participants standing alone. In a smaller group, the choices might be modified to religious, spiritual, agnostic/atheist, and other. The facilitation of a locogram may or may not include sharing. If the goal is to make a quick group choice, then sharing is likely unnecessary. But if the goal is to facilitate connection and explore shared experience, then sharing is likely to be helpful.

A locogram can also be used in teletherapy groups and online teaching sessions. This could be done in a variety of ways including using a poll feature, instructing participants to write their choice/preference in the chat box, or assigning numbers to each locogram choice and instructing participants to indicate their choice/preference by showing the number of fingers that corresponds with their choice. If the technology platform utilized allows for breakout rooms, the facilitator can initiate breakout rooms which will allow for participants to talk about their shared preference/choice. This method of adapting the locogram for teletherapy or online sessions is identical to how the floor check is modified for online groups. The floor check, a similar sociometry tool that evolved from the locogram, is outlined in the next section.

\subsection{Floor Checks}

The floor check is an experiential psychosocial process in the Relational Trauma Repair Model (RTR) created by Dayton $(2014,2015)$. It was inspired by the locogram process of using various places in the room for different options - the primary difference is that with the floor check the facilitator utilizes printed pieces of paper to label the options and offers a series of prompts each involving sharing. The floor check expands choice-making potential with increased options that transcend here-and-now prompts into past or future-oriented questions. The floor check expands the process with continual groupings and clustering which offer exponentially more chances for individual reflection, choice-making, group connection, education, and healing. The floor check was developed, based on evolving research on trauma, grief, mental health, addiction, and post-traumatic growth, to meet the pressing needs within addiction treatment centers which were faced with shorter group times, larger group sizes, varying degrees of client vulnerability, and therapists with different levels 
of psychodrama training. When employing the floor check process, it is advantageous to construct prompts or floor check options with research-based content. This can be done by simply choosing floor check options that correspond to the recognized symptomatology of a diagnosis or evidence-based research findings, theories, or practices (as described by Dayton, 2014, 2015). Thus, the floor check offers a psychoeducational and therapeutic healing avenue for addressing symptomatology in an engaging and dynamic process involving intrapsychic and interpersonal explorations. The multiple, focused prompts in a floor check sociometrically align participants based on the content while providing a progression of spontaneous connection and healing.

With each prompt, participants physically place themselves at the paper that corresponds to their preference or response. A floor check prompt results in group members clustering in small groups based on shared experience for verbal sharing about their choice. The process, similar to the locogram, provides a group-as-a-whole assessment but also cultivates a deepening of sharing and connection between group members in small groups. Floor checks "put healing in the hands of the process itself rather than exclusively in the hands of the therapists" (2015, p. 10) while empowering participants to become therapeutic agents for each other and quickly activating the mutual aid within a group (Giacomucci, 2019, 2020a, 2020b).

Once a floor check is put into action and group members have physically indicated their choices, they are invited to share with whoever is standing with them. When participants are standing alone at a choice, the facilitator simply directs them to join others nearby for sharing about their choice. This process creates sociometrically configured small groups, opening up opportunities for social-emotional learning and healing. Similar to the use of dyads or small groups, it allows the director to take a passive role in the process and move around the room listening or checking in on each cluster of clients. Floor checks are versatile and can be used in any group context with nearly any topic.

"box[Floor Check Video] starts"

The following video depicts the use of floor check with a live group.

http://www.phoenixtraumacenter.com/floor-check/

"box[Floor Check Video] ends"

Some useful clinical examples are listed below (more detailed prompts available in Dayton, 2014) including prompts following the previously described clinical map:

Feeling Floor Check-Anger; Sadness; Fear; Guilt/Shame; Happiness; Other

1. Which feeling best describes your experience today?

2. Which feeling most characterized your experience last week?

3. Which feeling do you most try to avoid?

4. Which feeling is hardest for you to tolerate in others?

5. Which feeling have you gotten better with? 
Relationships Floor Check-Family; Friends; Self; Groups/Communities; God/Higher Power; Other

1. Which relationship do you feel most supported by today?

2. Which relationship has the most conflict in it for you?

3. Which relationship has improved the most since you started therapy?

4. Which relationship would you like/need to work on today?

Defense Mechanisms Floor Check-Humor; Denial or Minimization; Rationalization or Intellectualization; Acting Out; Passive Aggression; Dissociation; Fight; Flight; Freeze; Other

1. Which defense do you feel you are most aware of using in your life today?

2. Which defense do you feel you have used the most in this group?

3. Which defense is most difficult for you to tolerate when someone else uses it?

4. Which defense do you no longer use as much as you used to?

Post-traumatic Stress Disorder Symptom Cluster Floor Check-Avoidance; Hyperarousal; Re-experiencing and Intrusions; Negative Mood and Cognitions

1. Which would you like to learn more about?

2. Which best describes how trauma has impacted you?

3. Which describes symptoms you previously experienced but now have effective coping skills for?

Domains of Post-traumatic Growth Floor Check-Personal Strength; Appreciation of Life; Relationships; Spiritual/Religious Growth; New Possibilities

1. Which domain do you feel you have grown in most?

2. Which domain do you feel you struggle with the most?

3. Which domain do you feel you could help someone in this group with?

The floor check can be modified for any group topic or theme that can be sectioned down into categories or choices. Other useful examples include the stages of change, the stages of grief, the tasks of resilience in ambiguous loss, mental health diagnoses, treatment themes, and strengths. This process is also valuable in educational spaces and can be used as an experiential teaching tool promoting reflection and the integration of concepts into personal experience (Giacomucci \& Skolnik, in-press). In social work education, it can be used with the social work core values, social work practice areas, or content from other theories (Giacomucci, 2019). The floor check is a holistic instrument that effectively warms people up physically, emotionally, socially, and to the chosen topic. It can be utilized alone as a group process or as a warm-up to another group process such as a psychodrama, art therapy piece, or writing process. 


\subsection{Step-in Sociometry}

The next sociometry process that we will explore is step-in sociometry-sometimes called circle of similarities. This experiential process is effective at quickly identifying shared experience or similarities in the group-as-a-whole (Archer, 2016). Buchanan (2016) notes that step-in sociometry is a newer addition to the sociometry toolbox in the 1970s from the New Games Movement (Fluegelman, 1976). Step-in sociometry is facilitated with the group stand in a circle. Prompts are offered with the instruction to step into the circle if you identify with the prompt. Prompts can be offered spontaneously, or the facilitator can ask participants to offer step-in prompts one at a time by going around the entire circle. It is helpful to encourage group members to make their statements broad and general rather than specific as it creates a more inclusive experience and prevents individuals from making statements so specific that they end up stepping in alone. If a prompt does result in one person alone in the circle, it is helpful to reframe the prompt in a more general way-for example, the prompt "I enjoy water color painting" could be generalized to "I enjoy creating art" which would result in more participants stepping in.

Either the facilitator offers prompts asking participants to physically step in if they identify, or group members take turns stepping in while making statements about themselves while others who identify also step in. Buchanan (2016) describes the latter as the democratic approach and the former as the totalitarian approach to stepin sociometry. This writer has discovered that the democratic approach is favorable in nearly all contexts. One exception where the totalitarian approach may be useful is when offering trauma-related prompts in a group where it could be harmful if the process goes too far into the trauma. The totalitarian approach would allow the facilitator to intentionally choose very broad trauma-related prompts that ensure the largest number of participants identify. In this context, the facilitator providing all of the prompts would contribute to group safety and containment. Another facilitation consideration is the facilitator's decision to participate in the process or not. The choice to participate or not, or participate in some rounds but not others, would come back to the facilitator's style, the goals for the group, the group population, and the group context. In some groups and with some prompts, it may be advantageous for the facilitator to participate which allows the group to connect with them while in other groups and with other prompts, it may be best for the director not to participate (Buchanan, 2016; Giacomucci, 2017).

In facilitating this process, it is also important to inform participants that they can choose whether they want to self-disclose or not on any given criteria as there may be prompts offered that some participants are willing to self-disclose while others may not be ready to do so (Buchanan, 2016). When a group member offers a prompt and other participants step in, it allows the group to visually see others who have a shared experience. Step-in sociometry makes the invisible connections and similarities within the group visible and conscious-thus increasing overall group connection and cohesion (Giacomucci et al., 2018). Once participants have stepped in, concretizing their connection to the prompt, the facilitator invites them to quietly 
acknowledge others who have stepped in then step back into the larger circle. This process seems to be appealing to introverted clients and young adults who find it to be an opportunity for peer identification without much verbal sharing (Giacomucci, 2017). Alternative ways of facilitating include inviting participants who have stepped into briefly share why they stepped in or to use sound or movement to express their connection to the prompt.

Step-in sociometry can be facilitated with multiple rounds, each with a larger theme, or as a single open-ended process without a theme. Both options have benefits. An open-ended step-in sociometry experience allows the group to choose any prompts which is likely to be revealing of the group's overall warm-up and allows the group to control the process (Archer, 2016). The method of using multiple rounds of step-in sociometry, each with themes, allows the facilitator to create a more directed and intentional group warm-up which may be most useful in clinical settings. When using multiple rounds of step-in sociometry, it is useful to follow the clinical map referenced previously.

"box[Step-in Sociometry Video] starts"

The following video depicts the use of step-in sociometry with a live group.

http://www.phoenixtraumacenter.com/step-in-sociometry/

"box[Step-in Sociometry Video] ends"

Below are some examples of different themes for step-in rounds:

\section{Group: Inpatient Addiction Treatment}

1. Step in and name something you like to do that is not related to your addiction.

2. Step in and name a consequence of your addiction (medical, emotional, social, legal, etc.).

3. Step in and share a hope or goal for your future in recovery from addiction.

\section{Group: Immigrant Families}

1. Step in and name something that is important to you about your family or culture.

2. Step in and label something that has been difficult for you related to immigration.

3. Step in and share a hope you have for you family going forward.

\section{Group: Grief and Loss Group for Parents}

1. Step in and share one thing that has helped you in your grief and loss.

2. Step in and share one difficult aspect related to your grief and loss.

3. Step in and share one goal for yourself and your family going forward in the grief process.

\section{Group: Hospital-Based Cancer Support Group}

1. Step in and share something about yourself beyond your medical condition.

2. Step in and share one difficult aspect of your cancer diagnosis.

3. Step in and name one goal for yourself going forward. 


\section{Group: High School Group}

1. Step in and share something about yourself.

2. Step in and share one thing you find difficult about high school.

3. Step in and share something you would like to accomplish by the end of high school.

\section{Group: New Social Work Graduate Students}

1. Step in and share one thing that influenced your decision to get a graduate degree in social work.

2. Step in and name one fear you have about the graduate program.

3. Step in and name a professional role that you would like to have in the future.

Step-in sociometry cultivates curiosity, connection, and inclusion with the group experience and can be adapted for any topic or group setting (Buchanan, 2016; Giacomucci, 2020a). Archer (2016) provides an in-depth exploration of various ways to adapt step-in sociometry with groups that consist of participants with physical limitations including group members who are visually impaired, wheelchair bound, or on crutches. In larger groups, or group spaces where creating a circle is not possible, the step-in sociometry process is often modified to utilize standing up or raising hands instead (Archer, 2016).

Similar to the previously described experiential sociometry processes, step-in sociometry can be used as a warm-up or as a group process on its own. Furthermore, the utilization of step-in sociometry for closure, processing, and integration after a psychodrama enactment or other group process has been described (Giacomucci, 2017). This may be most useful in the context of large groups or when time is limited and a quick, efficient method for sharing is called for.

\subsection{Hands-on-Shoulder Sociograms}

Section 5.5 of this book covered the theoretical aspects of the sociogram which was one of the first sociometry instruments that Moreno developed (Moreno, 1934). A sociogram shows the number of times group members choose each other based on specific criteria. Using pen-to-paper sociometric tests, Moreno collected the written choices of participants and drew complex sociograms to depict the distribution of choices within the group. The hands-on-shoulder sociogram, sometimes called an action sociogram, moves this process from paper into the room as an experiential process. The action sociogram makes the unseen choices within a group conscious, revealing the invisible web of attractions, and repulsions within a group (Korshak \& Shapiro, 2013). Hale (1981) refers to this as enhancing the sociometric consciousness of the group. While the previously described sociometry processes are all based on self-assessment, the sociogram is oriented on the assessment of others and the group's overall assessment of itself (Giacomucci, 2018a). 
The experiential sociogram process prompts participants to put their hand on the shoulder of one other group member based on a specific prompt, simultaneously revealing the distribution of choices and preference within the group. This process uses physical touch, so it is important to check in with the group about their comfort level with physical touch and obtain their consent - especially when working with trauma survivors. Sometimes, the aforementioned sociometry processes are used to explore comfort with physical touch such as a spectrogram, locogram, or step-in sociometry. If participants are not comfortable with others putting their hand on their shoulder, the process can be amended by having participants indicate their choices by touching their shoe to the shoe of another, standing next to another, pointing at their choices, or holding a scarf or string to indicate their choice (Hudgins \& Toscani, 2013; Olesen et al., 2017; Simmons, 2017). While some may find the experience of physical touch as uncomfortable or intolerable, others may find it soothing and comforting (Giacomucci et al., 2018). Moreno wrote of the power of physical touch and highlighted the practice wisdom from the field of nursing in this area (McIntosh, 2010; Moreno, 1972).

The sociogram often helps participants become aware of their own tendencies around choosing, waiting to be chosen, and prioritizing their first choice. When one has difficulty with making a choice, they are encouraged to choose with their intuition or to trust their tele. Once each participant has indicated their choice by putting their hand on the shoulder of one person in the room, the facilitator may offer a brief interpretation of the sociometric constellation-for example, noting prevalence of mutual choices, the equal distribution of choices, or the choices being highly concentrated with social stars and social isolates. The sociogram quickly depicts the distribution of criteria-specific social wealth within the group, thus highlighting the presence of tele and the sociodynamic effect. Then, participants are invited to share briefly with the person they chose about their reasoning for choosing them. This effectively increases interpersonal relationships within the group while enhancing overall group cohesion. Below are some examples of prompts tailored to different groups using the prescribed stages of the clinical map:

\section{Group: Veterans Support Group}

1. Place your hand on the shoulder of someone who you experienced take a significant step in their growth last month.

2. Place your hand on the shoulder of someone who has said something related to your identity as a veteran which was meaningful to you.

3. Place your hand on the shoulder of someone whose experience you would like to know more about.

4. Place your hand on the shoulder of someone who you could see yourself connecting with outside of group.

\section{Group: Substance Use Relapse Prevention Group}

1. Place your hand on the shoulder of someone who inspires you.

2. Place your hand on the shoulder of someone who has said something that worries you. 
3. Place your hand on the shoulder of someone who you could call if you had a craving to use drugs.

4. Place your hand on the shoulder of someone who you could call for relationship advice.

Each of the given examples demonstrates the use of hands-on-shoulder sociogram criteria that is reality-based and sociometric-based. Another option is to offer prompts that are surplus reality and psychodramatically oriented. The difference between reality-based sociometric prompts and surplus reality-based psychodrama prompts is depicted below:

\section{Sociometric Reality-Based Prompts:}

A. Place your hand on the shoulder of someone who you experience as courageous.

B. Place your hand on the shoulder of someone who you look up to as a role model.

C. Place your hand on the shoulder of someone who reminds you of yourself.

D. Place your hand on the shoulder of someone who you would call for spiritual guidance.

E. Place your hand on the shoulder of someone who you experience as motherly.

\section{Psychodramatic Surplus Reality-Based Prompts:}

A. Place your hand on the shoulder of someone who you would choose to play the role of your courage.

B. Place your hand on the shoulder of someone who you would choose to play the role of one of your role models.

C. Place your hand on the shoulder of someone who you would choose to play the role of yourself.

D. Place your hand on the shoulder of someone who you would choose to play the role of God.

E. Place your hand on the shoulder of someone who you would choose to play the role of your mother.

Some find that psychodramatic prompts provide more distance and thus more safety for group members when choosing and being chosen. Psychodramatic prompts also have the benefit of getting participants warmed up to choosing roles in a psychodrama. Many practitioners employ both sociometric and psychodramatic prompts in their use of sociograms in groups. Below are some examples of mixed prompts:

\section{Group: Depression Support}

1. Place your hand on the shoulder of someone who you would has helped you feel comfortable in the group.

2. Place your hand on the shoulder of someone who you would choose to play the role of your resilience.

3. Place your hand on the shoulder of someone who has helped you understand depression better. 
4. Place your hand on the shoulder of someone who you could call if your depression increased.

5. Place your hand on the shoulder of someone who you would choose to play the role of yourself in the future no longer experiencing depression.

\section{Group: Healthy Relationships for Couples}

1. Place your hand on the shoulder of someone whose sharing has helped you understand yourself better.

2. Place your hand on the shoulder of someone who you would choose to play the role of your willingness to work on your relationship.

3. Place your hand on the shoulder of someone whose love for their partner has inspired you.

4. Place your hand on the shoulder of someone who you would choose to play the role of your honesty or vulnerability.

5. Place your hand on the shoulder of someone who you would call if you needed support in the future.

While these are examples of prompts that could be offered in groups, it is important for the facilitator to remain attentive to the group process and to offer new prompts when needed to create a more inclusive experience. For example, if a facilitator notices that a participant repeatedly is unchosen, it would be important to spontaneously offer a new prompt that makes this person highly chosen. Group facilitators have a responsibility to be aware of the sociodynamic effect and to reverse its impact within the group to create an inclusive experience. While one may be unchosen in this group based on this criterion, they are almost certainly a social star when it comes to other criteria and in other groups. Korshak and Shapiro (2013) note that reversing the sharing in a sociogram is another method of reversing the sociodynamic effect. Rather than having participants share their reason for choosing another, the person chosen shares their experience of being chosen with the person choosing them. Moreno (2006) writes that the "essential reason for doing sociometric investigations is not just to make relationships visible and available for interpretation, but to reconstruct groups to maximize sociostasis and find some resolution to the problem of the unchosen and rejected" (p. 296).

At this point, it is important to note that of all of the experiential processes described in this chapter, the action sociogram has the most risk. Unless conducted attentively, spontaneously, and skillfully, the group may have a negative response to the hands-on-shoulder sociogram. This process is best utilized in higher functioning groups where participants are familiar with each other and have demonstrated a capacity for tolerating vulnerability and discomfort. 


\subsection{Circle of Strengths}

The circle of strengths is a safety structure that originates from the Therapeutic Spiral Model (TSM) (Hudgins \& Toscani, 2013). It is an experiential process of concretizing the collective strengths of the group and strongly compliments social work's strengths-based approach (Saleebey, 2012). Generally, this exercise begins with a large pile of scarves or other fabric in the center of the group room (though it can also be done with objects in the room or with pen/paper). As group members enter the room, the scarves spark their curiosity, playfulness, and creativity. One simple way of facilitating the circle of strengths is to have the group break up into dyads; if there are an odd number, the facilitator can join — this method of using dyads prevents anyone from being chosen last (Giacomucci et al., 2018). Participants are provided with psychoeducation on the importance of strengths and various types of strengths (intrapsychic, interpersonal, and transpersonal strengths). Participants are then asked to choose a scarf to represent a strength that they see in their partner and to present the strength to their partner one at a time while reminding their partner of examples of how they have demonstrated the strength. This provides a ritual for group members to practice healthy risks and vulnerability with each other based on positive criteria. The rest of the group witnesses the individual exchanges of strengths. As each strength is concretized (with scarves), they are placed on the floor of the group room, creating a large circle of strengths. This process can be repeated in new dyads or spontaneously between group members. The facilitator may choose to do one round in dyads, then a round where everyone identifies one of their own strengths, and finally a round where everyone concretizes a strength they experience in the group-as-a-whole.

The resulting circle of strengths on the floor serves as a conscious reminder of the individual and collective strengths within the group. They can be utilized by the facilitator later in the process if a group member becomes overwhelmed and might benefit from a reminder of their strengths. Symbolically, the circle is representative of the unity of the group and the ability of the collective group strengths to contain any participant's stories, experiences, trauma, or feelings. Logistically, the circle of strengths can serve as a stage for a future psychodrama in the group (Hudgins \& Toscani, 2013).

The exercise was initially developed through the process of having group members choose scarves to symbolize their own personal strengths that they bring to the group. As TSM became utilized around the world, especially in Asia, this process evolved to become more culturally sensitive and new methods developed including having group members choose scarves to concretize the strengths they see in each other (Hudgins \& Toscani, 2013). Novel methods for creating the circle of strengths continue to be developed including using short enactments of strengths, inviting group members to ask for reminders of specific strengths, and concretizing strengths of an organization or program. The experience of engaging in this process seems to significantly increase connections in the group, cultivate mutual aid, renegotiate 
one's sense of self, enhance group cohesion, and establish an "all in the same boat" mentality (Giacomucci, 2019, 2020b; Giacomucci et al., 2018; Shulman, 2010).

"box[Circle of Strengths Video] starts"

The following video depicts the use of circle of strengths with a live group.

http://www.phoenixtraumacenter.com/circle-of-strengths/

"box[Circle of Strengths Video] ends"

Some examples of statements from clients engaging in this process are provided below.

\section{Interpersonal Recognitions of Strengths}

- "I chose this for your courage because it is a strong and bold scarf. I have seen you demonstrate courage in the times you are vulnerable with us and in the stories you have shared about your childhood."

- "I chose this scarf to represent the compassion and kindness that I experience from you each time we are together. You always are friendly and are quick to offer support whenever anyone needs it-I know I can count on your support."

- "I picked this scarf to be your spirituality and the sense of purpose it gives you. It is clear that your spirituality is important to you and gives meaning to your life in the way you help others and maintain faith through uncertainty."

\section{Acknowledging Personal Strengths}

- "I chose this one to symbolize my resilience. Even though I have experienced hardship, I keep bouncing back and I survive no matter what."

- "This represents my relationship to my family. I believe family is the most important thing in life and they are always there to support me."

- "I chose this scarf for my ability to ground myself. I have learned breathing techniques and meditation which allow me to center myself internally even when things get chaotic."

\section{Strengths in the Group-as-a-Whole}

- "I chose this to represent our willingness to change. Everyone here demonstrates this strength each time you show up to group. Regardless of our failures or successes, we continue to be willing to change and grow."

- "I chose this colorful scarf to symbolize the diversity in this group. We all come from different walks of life and various backgrounds which gives us each a unique perspective that we bring to the group. I always learn new ways of looking at things because of you all."

- "I picked this scarf to represent the safety of this group. I usually don't trust people, but I know that this is a safe place and this group of people are worthy of my trust."

The circle of strength process can be quite powerful for participants and has the potential of tapping into strong emotions for some. As social workers, we often are 
working with traumatized, oppressed, and disenfranchised populations. The process of taking the time to recognize strengths can be incredibly restorative, especially for those that have been marginalized within society, dehumanized in their interpersonal relationships, or stuck in self-loathing. This process could be employed as a warm-up for a psychodrama or as its own group process. The circle of strengths can also be facilitated in online groups by having participants choose objects in their own room to concretize the strengths of others and themselves. It can also be adapted for use in community groups, supervision groups, student groups, and organizational settings to increase confidence and cohesion in a meaningful and strengths-based process.

\subsection{Conclusion}

The seven experiential sociometric processes outlined in this chapter provide social workers with essential strengths-based group tools that actively engage the groupas-a-whole. Each of these sociometric processes has their strengths and limitations, and is better equipped to meet different group work goals. For example, dyads, small groups, spectrograms, step-in sociometry, locograms, and floor checks are better equipped for early phases of a group to help initiate connection and sharing while the circle of strengths and hands-on-shoulder sociograms are best used once participants have already become more familiar with each other. Sociograms are superior compared to the other processes when it comes to uncovering the sociodynamic effect. Group choices are better decided with spectrograms, step-in sociometry, or locograms. Identifying where the group is at on the continuum of understanding or interest in a topic would best be done with spectrograms or locograms. Floor checks seem best equipped for psychoeducation and experiential teaching.

Each of these processes can be adapted for use with any topic, any population, any social work context, any type of group, any group size, and any phase of the group process. These experiential sociometry processes offer instruments for accessing the mutual aid that exists within all groups. Social workers are increasingly expected to facilitate group sessions in practice, while the provision of group work training has significantly decreased in graduate social work programs (Giacomucci, 2019; Skolnik-Basulto, 2016). These sociometry tools, with their countless applications, offer social workers with increased competencies to facilitate groups that keep participants engaged and active (Giacomucci \& Stone, 2019). The utilization of sociometry in clinical settings offers the social worker a system that is complimentary to both the unique philosophies of social work and social group work. The integration of sociometry into the repertoire of social work with groups gives social group work the backing to further differentiate itself from group psychology, group counseling, and sociology. 


\section{References}

Archer, M. (2016). Who, like me, loves to use the step-in circle? The Journal of Psychodrama, Sociometry, and Group Psychotherapy, 64(1), 79-82.

Buchanan, D. R. (2016). Practical applications of step-in sociometry: Increas-ing sociometric intelligence via self-disclosure and connection. Journal of Psychodrama, Sociometry, and Group Psychotherapy, 64, 71-78.

Chesner, A. (2020). Psychodrama and healing the traumatic wound. In A. Chesner \& S. Lykou (Eds.), Trauma in the creative and embodied therapies: When words are not enough (pp. 69-80). London: Routledge.

Courtois, C. A., \& Ford, J. D. (2016). Treatment of complex trauma: A sequenced, relationship-based approach. New York, NY: The Guildford Press.

Dayton, T. (2005). The Living Stage: A step-by-step guide to psychodrama, sociometry, and experiential group therapy. Deerfield, FL: Health Communications Inc.

Dayton, T. (2014). Relational Trauma Repair (RTR) Therapist's Guide (Revised ed.). New York, NY: Innerlook Inc.

Dayton, T. (2015). Neuro-psychodrama in the treatment of relational trauma: A strength-based, experiential model for healing PTSD. Deerfield Beach, FL: Health Communications Inc.

Fluegelman, A. (1976). The new games book. San Francisco, CA: New Games Foundation.

Freud, S. (1932). The dissection of the psychical personality. Standard edition (Vol. 22, pp. 67-80). London: Hogarth Press.

Giacomucci, S. (2017). The sociodrama of life or death: Young adults and addiction treatment. Journal of Psychodrama, Sociometry, and Group Psychotherapy, 65(1), 137-143. https://doi. org/10.12926/0731-1273-65.1.137.

Giacomucci, S. (2018a). Social work and sociometry: An integration of theory and clinical practice. The Pennsylvania Social Worker, 39(1), 14-16.

Giacomucci, S. (2018b). The trauma survivor's inner role atom: A clinical map for post-traumatic growth. Journal of Psychodrama, Sociometry, and Group Psychotherapy, 66(1), 115-129.

Giacomucci, S. (2019). Social group work in action: A sociometry, psychodrama, and experiential trauma therapy curriculum. Doctorate in Social Work (DSW) Dissertations. 124. https://reposi tory.upenn.edu/cgi/viewcontent.cgi?article=1128\&context=edissertations_sp2.

Giacomucci, S. (2020a). Addiction, traumatic loss, and guilt: A case study resolving grief through psychodrama and sociometric connections. The Arts in Psychotherapy, 67, 101627. https://doi. org/10.1016/j.aip.2019.101627.

Giacomucci, S. (2020b). Experiential sociometry in group work: Mutual aid for the group-asa-whole. Social Work with Groups, Advanced Online Publication. https://doi.org/10.1080/016 09513.2020.1747726.

Giacomucci, S., Gera, S., Briggs, D., \& Bass, K. (2018). Experiential Addiction Treatment: Creating Positive Connection through Sociometry and Therapeutic Spiral Model Safety Structures. Journal of Addiction and Addictive Disorders, 5, 17. http://doi.org/10.24966/AAD-7276/100017.

Giacomucci, S., \& Marquit, J. (2020). The effectiveness of trauma-focused psychodrama in the treatment of PTSD in inpatient substance abuse treatment. Frontiers in Psychology, 11, 896. https://dx.doi.org/10.3389\%2Ffpsyg.2020.00896.

Giacomucci, S., \& Skolnik, S. (in-press). The experiential social work educator: Integrating sociometry into the classroom environment. Journal of Teaching Social Work.

Giacomucci, S., \& Stone, A. M. (2019). Being in two places at once: Renegotiating traumatic experience through the surplus reality of psychodrama. Social Work with Groups, 42(3), 184-196. https://doi.org/10.1080/01609513.2018.1533913.

Hale, A. E. (1981). Conducting clinical sociometric explorations: A manual for psychodramatists and sociometrists. Roanoke, VA: Royal Publishing Company.

Hale, A. E. (2009). Moreno's sociometry: Exploring interpersonal connection. Group, 33(4), 347358. 
Herman, J. L. (1997). Trauma and recovery: The aftermath of violence-From domestic abuse to political terror. New York: Basic Books.

Hudgins, M. K., \& Toscani, F. (2013). Healing world trauma with the therapeutic spiral model: Stories from the frontlines. London: Jessica Kingsley Publishers.

Korshak, S. J., \& Shapiro, M. (2013). Choosing the unchosen: counteracting the sociodynamic effect using complementary sharing. The Journal of Psychodrama, Sociometry, and Group Psychotherapy, 61(1), 7-15.

Lawrence, C. (2015). The caring observer: Creating self-compassion through psychodrama. The Journal of Psychodrama, Sociometry, and Group Psychotherapy, 63(1), 65-72.

Levine, P. A. (2010). In an unspoken voice: How the body releases trauma and restores goodness. Berkeley, CA: North Atlantic Books.

McIntosh, W. (2010). Walking with Moreno: A historical journey of psychodrama and nursing. Australian and Aotearoa New Zealand Psychodrama Association Journal, 19, 30.

Moreno, J. L. (1934). Who shall survive? A new approach to the problems of human interrelations. Washington, D.C.: Nervous and Mental Disease Publishing Co.

Moreno, J. L. (1972). Psychodrama Volume 1 (4th ed.). New York: Beacon House.

Moreno, Z. (2006). The function of "tele" in human relations. In T. Horvatin \& E. Shreiber (Eds.), The quintessential Zerka: Writings by Zerka Toeman moreno on psychodrama, sociometry and group psychotherapy (pp. 289-301). New York, NY: Routledge.

Nieto, L. (2010). Look behind you: Using anti-oppression models to inform a protagonist's psychodrama. In E. Leveton (Ed.), Healing collective trauma using sociodrama and drama therapy (pp. 103-125). New York: Springer Publishing Company.

Olesen, J., Campbell, J., \& Gross, M. (2017). Using action methods to counter social isolation and shame among gay men. Journal of Gay \& Lesbian Social Services, 29(2), 91-108.

Saleebey, D. (2012). The strengths perspective in social work practice (6th ed.). Boston: Pearson Education.

Shulman, L. (2010). Dynamics and skills of group counseling. Belmont, CA: Cengage Learning.

Simmons, D. (2017). Implementing sociometry in a long-term care institutional setting for the elderly: Exploring social relationships and choices. The Journal of Psychodrama, Sociometry, and Group Psychotherapy, 65(1), 85-98.

Shapiro, F. (2018). Eye-movement desensitization and reprocessing (EMDR) therapy (3rd ed.). New York: Guilford Press.

Skolnik-Basulto, S. (2016). Coming together: A study of factors that influence social workers' connection to group work practice (Order No. 10758221). Available from ProQuest Dissertations \& Theses Global. (1988269052). Retrieved from https://proxy.library.upenn.edu/login? url=https://proxy.library.upenn.edu:2072/docview/1988269052?accountid=14707.

van der Kolk, B. A. (2014). The body keeps the score: Brain, mind, and body in the healing of trauma. New York: Viking Press.

Open Access This chapter is licensed under the terms of the Creative Commons Attribution 4.0 International License (http://creativecommons.org/licenses/by/4.0/), which permits use, sharing, adaptation, distribution and reproduction in any medium or format, as long as you give appropriate credit to the original author(s) and the source, provide a link to the Creative Commons license and indicate if changes were made.

The images or other third party material in this chapter are included in the chapter's Creative Commons license, unless indicated otherwise in a credit line to the material. If material is not included in the chapter's Creative Commons license and your intended use is not permitted by statutory regulation or exceeds the permitted use, you will need to obtain permission directly from the copyright holder. 